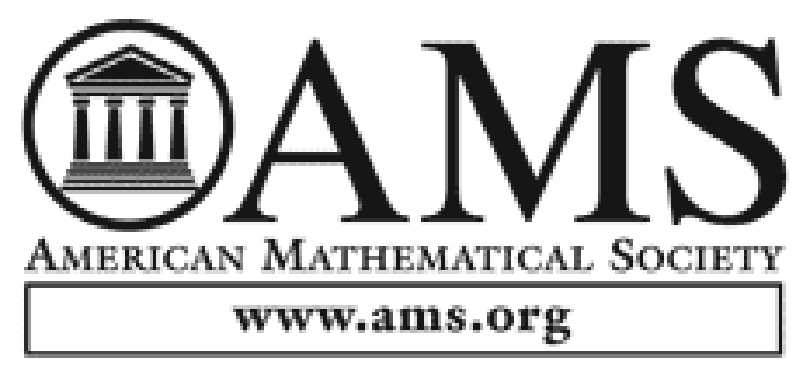

A Cohomological Class of Vector Bundles

Author(s): Rosa M. Miró-Roig

Source: Proceedings of the American Mathematical Society, Vol. 120, No. 4 (Apr., 1994), pp. 1003-1008

Published by: American Mathematical Society

Stable URL: http://www.jstor.org/stable/2160209

Accessed: 06/02/2009 07:48

Your use of the JSTOR archive indicates your acceptance of JSTOR's Terms and Conditions of Use, available at http://www.jstor.org/page/info/about/policies/terms.jsp. JSTOR's Terms and Conditions of Use provides, in part, that unless you have obtained prior permission, you may not download an entire issue of a journal or multiple copies of articles, and you may use content in the JSTOR archive only for your personal, non-commercial use.

Please contact the publisher regarding any further use of this work. Publisher contact information may be obtained at http://www.jstor.org/action/showPublisher?publisherCode=ams.

Each copy of any part of a JSTOR transmission must contain the same copyright notice that appears on the screen or printed page of such transmission.

JSTOR is a not-for-profit organization founded in 1995 to build trusted digital archives for scholarship. We work with the scholarly community to preserve their work and the materials they rely upon, and to build a common research platform that promotes the discovery and use of these resources. For more information about JSTOR, please contact support@jstor.org. 


\title{
A COHOMOLOGICAL CLASS OF VECTOR BUNDLES
}

\author{
ROSA M. MIRÓ-ROIG
}

(Communicated by Louis J. Ratliff, Jr.)

\begin{abstract}
The goal of this paper is to give a cohomological characterization of $F_{n, t}$, where $F_{n, t}:=\operatorname{Ker}\left((n+t ; n) \mathscr{Q}_{\mathbf{p}}(-t) \rightarrow \mathscr{Q}_{\mathbf{p}^{n}}\right)$.
\end{abstract}

\section{INTRODUCTION}

Fix an algebraically closed ground field $\mathbf{k}$ of characteristic zero. We set $S=\mathbf{k}\left[X_{0}, \ldots, X_{n}\right], m=\left(X_{0}, \ldots, X_{n}\right) \subset S$, and $\mathbf{P}^{n}=\operatorname{Proj}(S)$. For all positive integers $a, b$ with $a \geq b,((a ; b))$ will denote the binomial coefficient $((a ; b))=(a !) /(b !(a-b) !)$.

Choose a basis $v_{1}, \ldots, v_{a_{0}(n, t)}, a_{0}(n, t):=((n+t ; n))$ of $H^{0}\left(\mathbf{P}^{n} \mathscr{O}_{\mathbf{P}^{n}}(t)\right)$, $t>0$. Let $\Phi(n, t): \mathscr{O}_{\mathbf{P}^{n}} \rightarrow a_{0}(n, t) \mathscr{O}_{\mathbf{P}^{n}}(t)$ be the morphism defined by $\Phi(n, t)(c):=\left(c v_{1}, \ldots, c v_{a_{0}(n, t)}\right)$. Set $E_{n, t}:=\operatorname{Coker}(\Phi(n, t))$ and $F_{n, t}:=$ $E_{n, t}^{*}$ its dual. Note that $E_{n, t}, F_{n, t}$ are homogeneous and uniform vector bundles on $\mathbf{P}^{n}$. Furthermore, $E_{n, 1}=T_{\mathbf{P}^{n}}$ and $F_{n, 1}=\Omega_{\mathbf{P}^{n}}^{1}$, while $F_{n, t}$ and $E_{n, t}$ for $t>1$, for instance, are as defined in [G, MM]. In [G] they are used to give a new proof of the explicit Noether-Lefshetz Theorem and [MM] (see also [B]) stressed their importance for studying the Hartshorne-Rao module of a space curve.

However, not only the cotangent bundles $\Omega_{\mathrm{p}^{n}}^{1}$ are important but so are their exterior powers. So, we define $F_{n, t}^{r}:=\Lambda^{r} F_{n, t}$ for all $r \geq 1$ with the hope that they will also play an important role in the study of the cohomology groups of the ideal sheaf of closed subschemes of $\mathbf{P}^{n}$.

In $\S 1$ we will compute the cohomology groups and the order of $F_{n, t}^{r}$ and prove that $F_{n, t}$ are simple vector bundles on $\mathbf{P}^{n}$. In $\S 2$ we restrict our attention to the case $r=1$ and give the main theorem of this paper. Concretely, given a vector bundle $E$ on $\mathbf{P}^{n}$, we find sufficient conditions involving only suitably chosen cohomological groups in order that $E$ be the direct sum of $F_{n, t}$ and line bundles. Our essential tool will be the Beilinson spectral sequence.

Notation. For a coherent sheaf $F$ on $\mathbf{P}^{n}$ we use the abbreviation $s F=F \oplus$ $\cdots \oplus F$ for the $s$-fold direct sum of $F, H^{i} F(d)=H^{i}\left(\mathbf{P}^{n}, F \otimes \mathscr{O}_{\mathbf{P}^{n}}(d)\right)$, and $h^{i} F(d)=\operatorname{dim}_{k} H^{i} F(d)$.

Received by the editors December 13, 1991 and, in revised form, July 3, 1992.

1991 Mathematics Subject Classification. Primary 14F05.

The author was partially supported by DGICYT PB88-0224. 
First of all, we recall the definitions and basic facts that will be needed throughout this paper.

Definition 1.1 [E]. Let $E$ be a rank $r$ vector bundle on $\mathbf{P}^{n}$. We set $o(j)(E)=$ $\inf \left\{t \mid m^{t}\left(\bigoplus_{l} H^{j} E(l)\right)=0\right\}$. In other words, $o(j)(E)=r$ means that the morphism $H^{j} E(l) \rightarrow H^{j} E(l+r)$ given by multiplication by any homogeneous form of degree $r$ is zero. The order of $E$ is $o(E)=\max \{o(j)(E) \mid 1 \leq j \leq n-1\}$.

Proposition 1.2 [Ei, Proposition 1.1]. Let $E$ be a rank $r$ vector bundle on $\mathbf{P}^{n}$. Assume that $E$ is generated by its global sections. If $H^{n} E(-n-1) \neq 0$, then $E \cong \mathscr{O}_{\mathbf{P}^{n}} \oplus F$ for some vector bundle $F$ of rank $r-1$ on $\mathbf{P}^{n}$.

Beilinson Theorem [Be]. Let $F$ be a coherent sheaf on $\mathbf{P}^{n}$. There is a spectral sequence $E_{r}^{p q}$ with $E_{1}$-term $E_{1}^{p q}=H^{q}\left(\mathbf{P}^{n}, F(p)\right) \otimes \Omega_{\mathbf{P}^{n}}^{p}(-p)$ such that $E_{\infty}^{p q}=0$ for $p+q \neq 0$ and $\bigoplus_{p=0}^{n} E_{\infty}^{-p p}$ is the associated graded sheaf of a filtration of $F$.

Definition 1.3. For all integers $r \geq 1, n \geq 2$, set $a_{0}=a_{0}(n, r):=((r+n ; n))$ and $F_{n, r}:=\operatorname{Ker}\left(a_{0} \mathscr{O}_{\mathbf{P}^{n}}(-r) \rightarrow \mathscr{O}_{\mathbf{P}^{n}}\right) . F_{n, r}$ are homogeneous and uniform vector bundles of rank $a_{0}-1$ on $\mathbf{P}^{n}$. Note that $F_{n, 1}=\Omega_{\mathbf{P}^{n}}^{1}, F_{n, r} \mid \mathbf{P}^{n-1} \cong F_{n-1, r} \oplus$ $((n+r-1 ; r-1)) \mathscr{O}_{\mathbf{P}^{n-1}}(-r)$ where $\mathbf{P}^{n-1} \subset \mathbf{P}^{n}$ is a hyperplane, and the splitting type of $F_{n, r}$ is $\overbrace{-r-1, \ldots,-r-1}^{r \text { times }}, \underbrace{-r, \ldots,-r}_{a_{0}-r-1 \text { times }})$.

Proposition 1.4. For all integers $r \geq 1, n \geq 2$ the following hold:

(1) $H^{i} F_{n, r}(t)=0$ for all $t$, for all $i=1,2, \ldots, n-1$.

(2)

$$
h^{1} F_{n, r}(t)= \begin{cases}((t+n ; n)) & \text { if } 0 \leq t \leq r-1, \\ 0 & \text { otherwise. }\end{cases}
$$

(3) $F_{n, r}$ has order $r$.

(4) $F_{n, r}$ is $(r+1)$-regular. In particular, $F_{n, r}$ is globally generated for all $t \geq r+1$.

Proof. The proof follows from the exact sequence

$$
0 \rightarrow F_{n, r} \rightarrow a_{0} \mathscr{O}_{\mathbf{P}^{n}}(-r) \rightarrow \mathscr{O}_{\mathbf{P}^{n}} \rightarrow 0
$$

In [G], Green proves that $F_{n, r}(r)$ is 1-regular. We will compute the precise graded Betti numbers appearing in a minimal free resolution of $F_{n, r}(r)$.

Corollary 1.5. For all integers $r \geq 1, n \geq 2, F_{n, r}$ has a resolution of the following kind:

$$
\begin{aligned}
& 0 \rightarrow a_{n}(n, r) \mathscr{O}_{\mathbf{P}^{n}}(-n-r) \rightarrow \cdots \\
& \quad \rightarrow \cdots \rightarrow a_{i}(n, r) \mathscr{O}_{\mathbf{P}^{n}}(-r-i) \\
&\quad \cdots, r) \mathscr{O}_{\mathbf{P}^{n}}(-r-2) \rightarrow a_{1}(n, r) \mathscr{O}_{\mathbf{P}^{n}}(-r-1) \rightarrow F_{n, r} \rightarrow 0
\end{aligned}
$$

where $a_{i}(n, r)=\sum_{j=1}^{i}(-1)^{j-1}((n+j ; j)) a_{i-j}(n, r)+(-1)^{i}((n+r+i ; n))$. Proof. The proof follows after a tedious computation. 
Definition 1.6. For all integers $r, p \geq 1, n \geq 2$, we define $F_{n, r}^{p}$ as the $p$ th exterior power of the vector bundle $F_{n, r}$; thus, $F_{n, r}^{p}:=\Lambda^{p} F_{n, r}$.

Fact 1.7. Let $0 \rightarrow E \rightarrow F \rightarrow G \rightarrow 0$ be an exact sequence of vector bundles. Then we have the following exact sequences involving alternating and symmetric powers (Eagon-Northcott complexes):

$$
0 \rightarrow \Lambda^{q} E \rightarrow \Lambda^{q} F \rightarrow \Lambda^{q-1} F \otimes G \rightarrow \cdots \rightarrow F \otimes S^{q-1} G \rightarrow S^{q} G \rightarrow 0
$$

and

$$
0 \rightarrow S^{q} E \rightarrow S^{q-1} E \otimes F \rightarrow \cdots \rightarrow E \otimes \Lambda^{q-1} F \rightarrow \Lambda^{q} F \rightarrow \Lambda^{q} G \rightarrow 0 .
$$

Proposition 1.8. For all integers $r \geq 1, n \geq 2$, and $a_{0}-1 \geq p \geq 2$, the following hold:

(1) $F_{n, r}^{p}$ is $p(r+1)$-regular. In particular, $F_{n, r}^{p}$ is globally generated for all $t \geq p(r+1)$.

(2) $H^{i} F_{n, r}^{p}(t)=0$ for all $t$, for all $i=p+1, \ldots, n-1$.

(3) $F_{n, r}^{p}$ has order less or equal to $r+p-2$.

Proof. (1) By Proposition 1.4, $F_{n, r}$ is $(r+1)$-regular. Since we are working in characteristic zero, $F_{n, r}^{p}:=\Lambda^{p} F_{n, r}$ are direct summands of the $p$-fold tensor product $T^{p} F_{n, r}$ of $F_{n, r}$ which are $p(r+1)$-regular.

From the $p$ th exterior power of the exact sequence $(*)$ taking into account Fact 1.7 we get the exact sequence

$$
0 \rightarrow F_{n, r}^{p}(p r) \rightarrow\left(\left(a_{0} ; p\right)\right) \mathscr{O}_{\mathbf{P}^{n}} \rightarrow F_{n, r}^{p-1}(p r) \rightarrow 0 .
$$

Now, (2) and (3) easily follows from the exact sequence (**).

It seems not easy to decide whether the vector bundles $F_{n, r}$ are stable or not, however, we can prove that they are at least simple.

Proposition 1.9. For all integers $r \geq 1, n \geq 2, F_{n, r}$ are simple.

Proof. We tensor the exact sequence

$$
0 \rightarrow \mathscr{O}_{\mathbf{P}^{n}} \rightarrow a_{0} \mathscr{O}_{\mathbf{P}^{n}}(r) \rightarrow F_{n, r}^{*} \rightarrow 0
$$

with $F_{n, r}$ and obtain

$$
0 \rightarrow F_{n, r} \rightarrow a_{0} F_{n, r}(r) \rightarrow F_{n, r} \otimes F_{n, r}^{*} \rightarrow 0 .
$$

The cohomology sequence is as follows:

$$
\begin{aligned}
\cdots & \rightarrow H^{0}\left(\mathbf{P}^{n}, a_{0} F_{n, r}(r)\right) \rightarrow H^{0}\left(\mathbf{P}^{n}, F_{n, r} \otimes F_{n, r}^{*}\right) \\
& \rightarrow H^{1}\left(\mathbf{P}^{n}, F_{n, r}\right) \rightarrow H^{1}\left(\mathbf{P}^{n}, a_{0} F_{n, r}(r)\right) \rightarrow .
\end{aligned}
$$

From Proposition 1.4, it follows that $H^{0}\left(\mathbf{P}^{n}, F_{n, r} \otimes F_{n, r}^{*}\right) \cong H^{1}\left(\mathbf{P}^{n}, F_{n, r}\right) \cong k$. Thus, $F_{n, r}$ is simple.

Now, using Beilinson's theorem, we will give sufficient conditions involving only a finite number of suitably chosen cohomology groups in order that a vector bundle $E$ on $\mathbf{P}^{n}$ be the direct sum of $F_{n, r}$ and line bundles. 
Theorem 2.1. Let $E$ be a rank $\rho$ vector bundle on $\mathbf{P}^{n}$ such that:

(1) $H^{i} E(t)=0$ for all $t$, for all $i=2, \ldots, n-1$;

(2)

$$
h^{1} E(t)= \begin{cases}((t+r+n ; m)) & \text { if }-r \leq t \leq-1, \\ 0 & \text { otherwise }\end{cases}
$$

(3) $H^{0} E=0$.

Then $E$ has order $r$ and $E \cong F_{n, r}(r) \oplus$ (line bundles).

Proof. From Definition 1.1 it follows that the order of $E$ is $r$. Let $t=$ $\max \left\{l \mid H^{n} E(l) \neq 0\right\}$. If $t \geq-n$, then $E(t+n+1)$ is generated by its global sections and $H^{n} E(t) \neq 0$. Hence, by Proposition 1.2, $E \cong E_{0} \oplus \mathscr{O}_{\mathbf{p}}(-t-1-n)$ for some $(\rho-1)$-vector bundle $E_{0}$ on $\mathbf{P}^{n}$. Repeating this argument we may assume that $E \cong F \oplus\left(\bigoplus_{i} \mathcal{O}_{\mathbf{P}^{n}}\left(a_{i}\right)\right)$ where $-t-n-1 \leq a_{1}<0$ and $F$ is a vector bundle on $\mathbf{P}^{n}$ such that:

(1) $H^{0} F=0$;

(2) $H^{i} F(t)=0$ for all $t$, for all $i=2, \ldots, n-1$;

(3) $H^{n} F(t)=0$ for all $t \geq-n$;

(4)

$$
h^{1} F_{n, r}(t)= \begin{cases}((t+r+n ; n)) & \text { if }-r \leq t \leq-1, \\ 0 & \text { otherwise. }\end{cases}
$$

To end the proof it is enough to see that $F \cong F_{n, r}(r)$. We apply Beilinson's spectral sequence with $E_{1}$-terms $E_{1}^{p q}=H^{q}\left(\mathbf{P}^{n}, F(p)\right) \otimes \Omega_{\mathbf{P}^{n}}^{p}(-p)$. The diagram of the $E_{1}$-terms is as follows:

$$
\left.\begin{array}{ccccc}
0 & \cdots & 0 & 0 & 0 \\
\cdot & \cdots & \cdot & \cdot & \cdot \\
\cdot & \cdots & \cdot & \cdot & \cdot \\
\dot{0} & \cdots & \dot{0} & 0 & 0 \\
\Omega^{n}(n)^{h^{1} F(-n)} & \cdots & \Omega^{2}(2)^{h^{1} F(-2)} & \Omega^{1}(1)^{h^{1} F(-1)} & 0 \\
0 & \cdots & 0 & 0 & 0
\end{array}\right\}_{p}
$$

Since $E_{2}^{p q}=E_{\infty}$, the only nonzero row is exact with only one exception $\Omega_{\mathbf{P}^{n}}^{1}(1)^{h^{1} F(-1)}$ where the cokernel is $F$. So, we have the exact sequence

$$
\begin{aligned}
0 & \rightarrow \Omega_{\mathbf{P}^{n}}^{n}(n)^{h^{1} F(-n)} \rightarrow \cdots \rightarrow \Omega_{\mathbf{P}^{n}}^{2}(2)^{h^{1} F(-2)} \\
& \rightarrow \Omega_{\mathbf{P}^{n}}^{1}(1)^{h^{1} F(-1)} \rightarrow F \rightarrow 0 .
\end{aligned}
$$

In particular, we get that $c_{i}(F)=c_{i}\left(F_{n, r}(r)\right)$ for $i=1, \ldots, n$; and $\operatorname{rk}(F)=$ $\operatorname{rk}\left(F_{n, r}(r)\right)$. Hence, in order to prove that $F$ and $F_{n, r}(r)$ are isomorphic it is enough to see that there is a monomorphism between $F_{n, r}$ and $F$. First of all, note that applying $\operatorname{Hom}(\cdot, F)$ to the exact sequence

$$
0 \rightarrow F_{n, r}(r) \rightarrow a_{0} \mathscr{O}_{\mathbf{p}^{n}} \rightarrow \mathscr{O}_{\mathbf{p}^{n}}(r) \rightarrow 0,
$$

we get the exact sequence

$$
\begin{aligned}
0 & \rightarrow \operatorname{Hom}\left(\mathscr{O}_{\mathbf{P}^{n}}(r), F\right) \rightarrow a_{0} \operatorname{Hom}\left(\mathscr{O}_{\mathbf{P}^{n}}, F\right) \rightarrow \operatorname{Hom}\left(F_{n, r}(r), F\right) \\
& \rightarrow \operatorname{Ext}^{1}\left(\mathscr{O}_{\mathbf{P}^{n}}(r), F\right) \rightarrow a_{0} \operatorname{Ext}^{1}\left(\mathscr{O}_{\mathbf{P}^{n}}, F\right) \rightarrow \cdots .
\end{aligned}
$$


Since $\operatorname{Hom}\left(\mathscr{O}_{\mathbf{P}^{n}}, F\right)=H^{0}\left(\mathbf{P}^{n}, F\right)=0$ and $\operatorname{Ext}^{1}\left(\mathscr{O}_{\mathbf{P}^{n}}, F\right)=H^{1}\left(\mathbf{P}^{n}, F\right)=0$, we conclude that $\operatorname{Hom}\left(F_{n, r}(r), F\right) \cong \operatorname{Ext}^{1}\left(\mathscr{O}_{\mathbf{P}^{n}}(r), F\right) \cong\left(\mathbf{P}^{n}, F(-r)\right) \cong k$. Similarly, applying $\operatorname{Hom}\left(\cdot, F_{n, r}(r)\right)$ to the exact sequence $(* * *)$ we get that $\operatorname{Hom}\left(F, F_{n, r}(r)\right) \neq 0$. Now, we choose a nontrivial morphism $\Phi: F_{n, r}(r) \rightarrow F$ and $\Psi: F \rightarrow F_{n, r}(r)$ and consider the composition $\Psi \Phi: F_{n, r}(r) \rightarrow F_{n, r}(r)$. Since $F_{n, r}(r)$ are simple, we have $\Psi \Phi=c \operatorname{Id}_{F_{n, r}(r)}$ for some $c \in k$.

Claim. $c \neq 0$.

Since $c$ is a nonzero constant, we conclude that $\Phi$ is a monomorphism, which gives the desired result.

Proof of the Claim. Assume that $\Psi \Phi=0$. Set $a_{i}=h^{1} F(-i)$. We have the exact sequences:

$$
0 \rightarrow \Omega_{\mathbf{P}^{n}}^{n}(n)^{a_{n}} \underset{\rho_{n}}{\longrightarrow} \cdots \underset{\rho_{3}}{\longrightarrow} \Omega_{\mathbf{P}^{n}}^{2}(2)^{a_{2}} \underset{\rho_{2}}{\longrightarrow} \Omega_{\mathbf{P}^{n}}^{1}(1)^{a_{1}} \underset{\beta}{\longrightarrow} F_{n, r}(r) \longrightarrow 0
$$

and

$$
0 \rightarrow \Omega_{\mathbf{P}^{n}}^{n}(n)^{a_{n}} \rightarrow \cdots \rightarrow \Omega_{\mathbf{P}^{n}}^{2}(2)^{a_{2}} \rightarrow \Omega_{\mathbf{P}^{n}}^{1}(1)^{a_{1}} \underset{\gamma}{\longrightarrow} F \rightarrow 0 .
$$

Cutting (2) into short exact sequences, we prove that the morphism $\Phi \beta$ can be lifted to a nontrivial morphism $f: \Omega_{\mathbf{P}^{n}}^{1}(1)^{a_{1}} \rightarrow \Omega_{\mathbf{P}^{n}}^{1}(1)^{a_{1}}$ in order that the following square commutes:

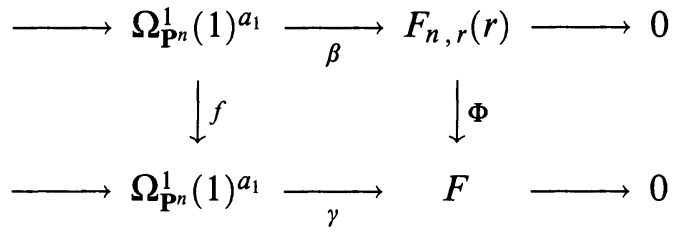

In the same way we get a commutative diagram:

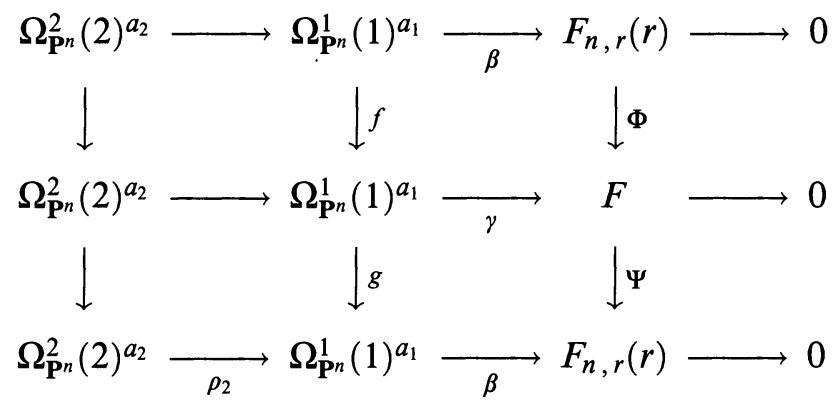

Hence, we have $0=\Psi \Phi \beta=\beta g f$. Therefore, $\operatorname{Im}(g f) \subset \operatorname{Ker}(\beta)=\operatorname{Im}\left(\rho_{2}\right)$ and $g f$ can be lifted to a nontrivial morphism $h: \Omega_{\mathbf{p}^{n}}^{1}(1)^{a_{1}} \rightarrow \operatorname{Im} g\left(\rho_{2}\right)$. Finally, applying the functor $\operatorname{Hom}\left(\Omega_{\mathbf{P}^{n}}^{1}(1)^{a_{1}}, \cdot\right)$ to the short exact sequence

$$
0 \longrightarrow \operatorname{Ker}\left(\rho_{2}\right) \hookrightarrow \Omega_{\mathbf{P}^{n}}^{2}(2)^{a_{2}} \underset{\rho_{2}}{\longrightarrow} \operatorname{Im} g\left(\rho_{2}\right)=\operatorname{Ker}(\beta) \rightarrow 0
$$

and taking into account that $\operatorname{Ext}^{1}\left(\Omega_{\mathbf{p}^{n}}^{1}(1)^{a_{1}}, \operatorname{Ker}\left(\rho_{2}\right)\right)=0$, we get that $h$ and, hence, $f g$ can be lifted to a nontrivial morphism $\Omega_{\mathbf{P}^{n}}^{1}(1)^{a_{1}} \rightarrow \Omega_{\mathbf{P}^{n}}^{2}(2)^{a_{2}}$. This is a contradiction because $\operatorname{Hom}\left(\Omega_{\mathbf{P}^{n}}^{1}(1), \Omega_{\mathbf{P}^{n}}^{2}(2)\right)=0$. 
As a corollary, we have the following well-known result:

Corollary 2.2. Let $E$ be a rank $\rho$ vector bundle on $\mathbf{P}^{n}$ such that $H^{i} E(*)=0$ for $0<i<n$ with the only exception $h^{1} E(-1)=1$. Then, $E \cong \Omega^{1}(1) \oplus$ (line-bundles).

Proof. Set $t=\min \left\{l \mid H^{0} E(l) \neq 0\right\}$. If $t \leq 0$, then $H^{i} E(t-i-1)=0$ for $0 \leq i<n$. Hence, by [AO, Theorem 2], $E \cong F_{1} \oplus \mathscr{O}(-t)^{h^{0} F(t)}$ where $F_{1}$ is a locally free sheaf on $\mathbf{P}^{n}$ such that $H^{i} F_{1}(*)=0$ for $0<i<n$ with only exception $h^{1} F_{1}(-1)=1$ and $\min \left\{l \mid H^{0} E(l) \neq 0\right\}<\min \left\{l \mid H^{0} F_{1}(l) \neq 0\right\}$. Repeating this process we may assume that $E \cong F \oplus$ (line-bundles) where $F$ is a locally free sheaf on $\mathbf{P}^{n}$ such that $H^{i} F(*)=0$ for $0<i<n$ with only exception $h^{1} F(-1)=1$ and $\min \left\{l \mid H^{0} F(l) \neq 0\right\}>0$. Now, applying Theorem 2.1, we have $F \cong \Omega^{1}(1) \oplus$ (line-bundles), which gives the desired result.

Question 2.4. Given a vector bundle $E$ on $\mathbf{P}^{n}$, are there sufficient conditions involving only a finite number of suitably chosen cohomology groups in order that the vector bundle $E$ be the direct sum of $F_{n, r}^{p}$ and line bundles?

\section{REFERENCES}

[AO] V. Ancona and G. Ottaviani, Some applications of Beilinson theorem to sheaves of varieties, preprint, 1989.

[B] E. Ballico, Life and death of a cohomology class, J. Algebra 141 (1991), 265-275.

[Be] A. Beilinson, Coherent sheaves on $\mathbf{P}^{n}$ and problems of linear algebra, Funct. Anal. Appl. 12 (1978), 214-216.

[E] Ph. Ellia, Ordres et cohomologie des fibrés de rang deux sur l'espace projectif, preprint, 1987.

[Ei] L. Ein, An analogue of Max Noether's theorem, Duke Math. J. 52 (1985), 687-706.

[G] M. Green, A new proof of the explicit Noether-Lefschetz theorem, J. Differential Geom. 27 (1988), 155-159.

[MM] J. Migilore and R. M. Miró-Roig, On k-Buchsbaum curves, Comm. Algebra 18 (1990), 2403-2422.

[OSS] C. Okonek, M. Schneider, and H. Spindler, Vector bundles on complex projective spaces, Birkhäuser, Basel, 1980.

Departmento Matemáticas, Facultad de Ciencias, Universidad de Zaragoza, 50009 ZARAGOZA, SPAIN

E-mail address: Miro@cc.unizar.es

Current address: Departmento Algebra y Geometria, Facultad Matematicas, Universidad Barcelona, 08007 Barcelona, Spain 